\title{
Assessment of population genetic structure in the arbovirus vector midge, Culicoides brevitarsis (Diptera: Ceratopogonidae), using multi-locus DNA microsatellites
}

\author{
Maria G Onyango ${ }^{1,2}$, Nigel W Beebe ${ }^{3,4}$, David Gopurenko ${ }^{5,6}$, Glenn Bellis ${ }^{7}$, Adrian Nicholas ${ }^{6}$, Moses Ogugo $^{8}$,
} Appolinaire Djikeng ${ }^{8,9}$, Steve Kemp $^{8}$, Peter J Walker ${ }^{1}$ and Jean-Bernard Duchemin ${ }^{1 *}$

\begin{abstract}
Bluetongue virus (BTV) is a major pathogen of ruminants that is transmitted by biting midges (Culicoides spp.). Australian BTV serotypes have origins in Asia and are distributed across the continent into two distinct episystems, one in the north and another in the east. Culicoides brevitarsis is the major vector of BTV in Australia and is distributed across the entire geographic range of the virus. Here, we describe the isolation and use of DNA microsatellites and gauge their ability to determine population genetic connectivity of C. brevitarsis within Australia and with countries to the north. Eleven DNA microsatellite markers were isolated using a novel genomic enrichment method and identified as useful for genetic analyses of sampled populations in Australia, northern Papua New Guinea (PNG) and Timor-Leste. Significant $(P<0.05)$ population genetic subdivision was observed between all paired regions, though the highest levels of genetic sub-division involved pair-wise tests with PNG (PNG vs. Australia $\left(F_{S T}=0.120\right)$ and PNG vs. Timor-Leste $\left(F_{S T}=0.095\right)$ ). Analysis of multi-locus allelic distributions using STRUCTURE identified a most probable two-cluster population model, which separated PNG specimens from a cluster containing specimens from Timor-Leste and Australia. The source of incursions of this species in Australia is more likely to be Timor-Leste than PNG. Future incursions of BTV positive C. brevitarsis into Australia may be genetically identified to their source populations using these microsatellite loci. The vector's panmictic genetic structure within Australia cannot explain the differential geographic distribution of BTV serotypes.
\end{abstract}

\section{Introduction}

Bluetongue (BT) is an economically important viral disease throughout tropical and temperate regions of the world, posing a threat to the livestock industries, through production losses and negative impacts on trade [1]. The disease affects primarily sheep and goats. Cattle can also be infected but rarely show signs of disease [2]. Biting midges (Culicoides spp.) are vectors of bluetongue virus (BTV). In Australia, C. actoni Smith, C. brevitarsis Kieffer, C. fulvus Sen and Das Gupta are proven vectors of BTV and several others including C. brevipalpis Delfinado, $C$. dumdumi Sen and Das Gupta C. oxystoma Kieffer, C.

\footnotetext{
* Correspondence: Jean-Bernard.Duchemin@csiro.au

${ }^{1}$ CSIRO Health \& Biosecurity Australian Animal Health Laboratory, 5

Portalington Road, Geelong, Victoria 3220, Australia

Full list of author information is available at the end of the article
}

peregrinus Kieffer and $C$. wadai Kitaoka are regarded as potential vectors $[3,4]$. Of these species, $C$. brevitarsis is the most widely distributed throughout northern and eastern parts of the continent [5,6], and is considered to be the major vector, employing cattle and buffalo dung as breeding sites $[4,7]$.

BTV appears to have been introduced to Australia from Southeast Asia on multiple occasions by infected wind-borne vectors [8,9]. Indeed, 10 of the 26 known BTV serotypes have been detected in Australia through intensive surveillance during the past 30 years and there is evidence that at least four of these serotypes were introduced since the surveillance programme commenced [10]. The absence of clinical bluetongue disease in Australia, despite evidence of widespread infection in cattle, has been attributed to the limited distribution of 
C. brevitarsis to non-sheep breeding regions, primarily in the south of the continent and the relatively low pathogenicity of Australian BTV serotypes. Surveillance has indicated that the distribution of BTV serotypes in Australia is asymmetric with all 10 serotypes detected in the far northern region and only two serotypes (BTV-1 and BTV-21) enzootic in the southern portions of the eastern states. The factors influencing the distribution of serotypes are unknown and there is concern that introductions of exotic BTV strains from Southeast Asia via windborne Culicoides could destabilize the current situation [9]. A recent study of long-distance dispersal of Culicoides midges using an aerial migration model, indicated that migration of Culicoides into northern Australia from Timor-Leste (TL) and Papua New Guinea (PNG) is possible with Timor considered the most likely source of incursions [11]. Recent phylogeographic analyses [12,13] generally support those contentions and further indicate C. brevitarsis likely entered Australia and PNG separately from independent southeast Asian sources, in recent historical times [13]. Results of those prior genetic studies were based on analyses of a single maternally inherited gene and are potentially biased by a variety of evolutionary, demographic and sampling processes [14,15]. Additional population genetic analyses using multiple independent loci are needed to test hypotheses concerning the origins of recent arrivals of midge species in Australia.

The first aim of this study was to develop a technical workflow for identifying DNA microsatellite markers de novo from small organisms such as Culicoides from which limited quantities of genomic DNA can be extracted. The second aim was to identify and compare allelic diversity of microsatellite loci among C. brevitarsis in Australia and neighbouring countries (PNG and $\mathrm{TL}$ ) that are suspected sources of Culicoides spp. entering Australia. In this latter aim, we also sought to determine the levels of population genetic connectivity among the regions and infer the likely source(s) of midges in Australia during historical and current times.

\section{Materials and methods}

\section{Insect sampling and DNA preparation}

A total of 141 samples were collected using light trap or sweep net, preserved in $70 \%$ ethanol and identified from sites in the Northern Territory (NT), Queensland (QLD), New South Wales (NSW), PNG and TL (Figure 1A) as described by Gopurenko et al. [13]. Species identification was verified using genetic methods reported in $[16,17]$ to ensure morphologically related species of Culicoides were not included in analyses. After species identification, total genomic DNA was extracted from single specimens of $C$. brevitarsis using the DNeasy blood and tissue kit (Qiagen) according to the manufacturer's protocol and by a non-destructive genomic DNA extraction method [16]. The genomic DNA was quantified using a Qubit fluorometer (Life Technologies, Invitrogen).

\section{Whole genome amplification of $C$. brevitarsis}

To improve the amount of genomic DNA yield from singleton Culicoides for downstream manipulations, multiple displacement amplification-based (MDA) whole genome amplification (WGA) using Repli-g ultrafast mini kit (QIAGEN) was conducted on specimen DNA according to the manufacturers' protocol. To assess the differences in yield of amplification from a range of starting amounts of genomic DNA and according to two different denaturing procedures, $10.7 \mathrm{ng}$ and $0.215 \mathrm{ng}$ of DNA were denatured either by heat $\left(95^{\circ} \mathrm{C}\right.$ for $\left.3 \mathrm{~min}\right)$ and or by adding denaturing solution (buffer D1; QIAGEN) and then amplified using the REPLI-g ultrafast mini kit at $30{ }^{\circ} \mathrm{C}$ for $1.5 \mathrm{~h}$ followed by polymerase inactivation at $65{ }^{\circ} \mathrm{C}$ for $3 \mathrm{~min}$. Water was used as a negative control. The resulting DNA was quantified using a Qubit fluorometer and qualitatively assessed by electrophoresis (1\% agarose gel (Invitrogen) at $7.40 \mathrm{~V} / \mathrm{cm}$ ) in the presence of $1 \mathrm{~kb}$ DNA ladder and staining/UV visualisation.

\section{Quality check of whole genome amplified DNA}

To evaluate the quality of the whole genome amplified DNA, the products of the previous amplification (together with the negative control) were used as templates to PCR amplify a 600 bp housekeeper gene (actin). Each $20 \mu \mathrm{L}$ reaction included $1 \mu \mathrm{L}$ template DNA (approximately $20 \mathrm{ng}$ ), BSA $(400 \mathrm{ng} / \mu \mathrm{L}), 5 \mu \mathrm{m}$ each forward and reverse primer (Act-2 F and Act-8R) [18] and $15 \mu \mathrm{L}$ Platinum PCR supermix high fidelity (Life Technologies). The cycling profile was $94{ }^{\circ} \mathrm{C}$ for $2 \mathrm{~min}$, 35 cycles of $94{ }^{\circ} \mathrm{C}$ for $30 \mathrm{~s}, 58{ }^{\circ} \mathrm{C}$ for $30 \mathrm{~s}, 72{ }^{\circ} \mathrm{C}$ for $30 \mathrm{~s}$ and a final elongation at $72{ }^{\circ} \mathrm{C}$ for $5 \mathrm{~min}$ [18]. The PCR products were assessed for size by electrophoresis as described earlier.

\section{Isolation and screening of microsatellite repeats and primer design}

To isolate DNA microsatellite markers, $1 \mu \mathrm{L}$ of a pooled specimen DNA ( $n=7$ New South Wales, $n=8$ Northern Territory) was whole genome-amplified. The amplified whole genomic DNA was sequenced on $1 / 8$ plate Roche 454 Genome Sequencer FLX plus by an external contractor (Macrogen, Geumchun-gu, Seoul). The raw sequence reads of amplified whole genomic DNA were screened directly for di-, tri- and tetranucleotides using MSATCOMMANDER v0.8.2 [19]. Primers were designed to the flanking regions of the microsatellite repeats using the Primer 3 program [20]. The primers were checked for similarities amongst each other and were blasted against the NCBI database using BLASTN to determine if the 


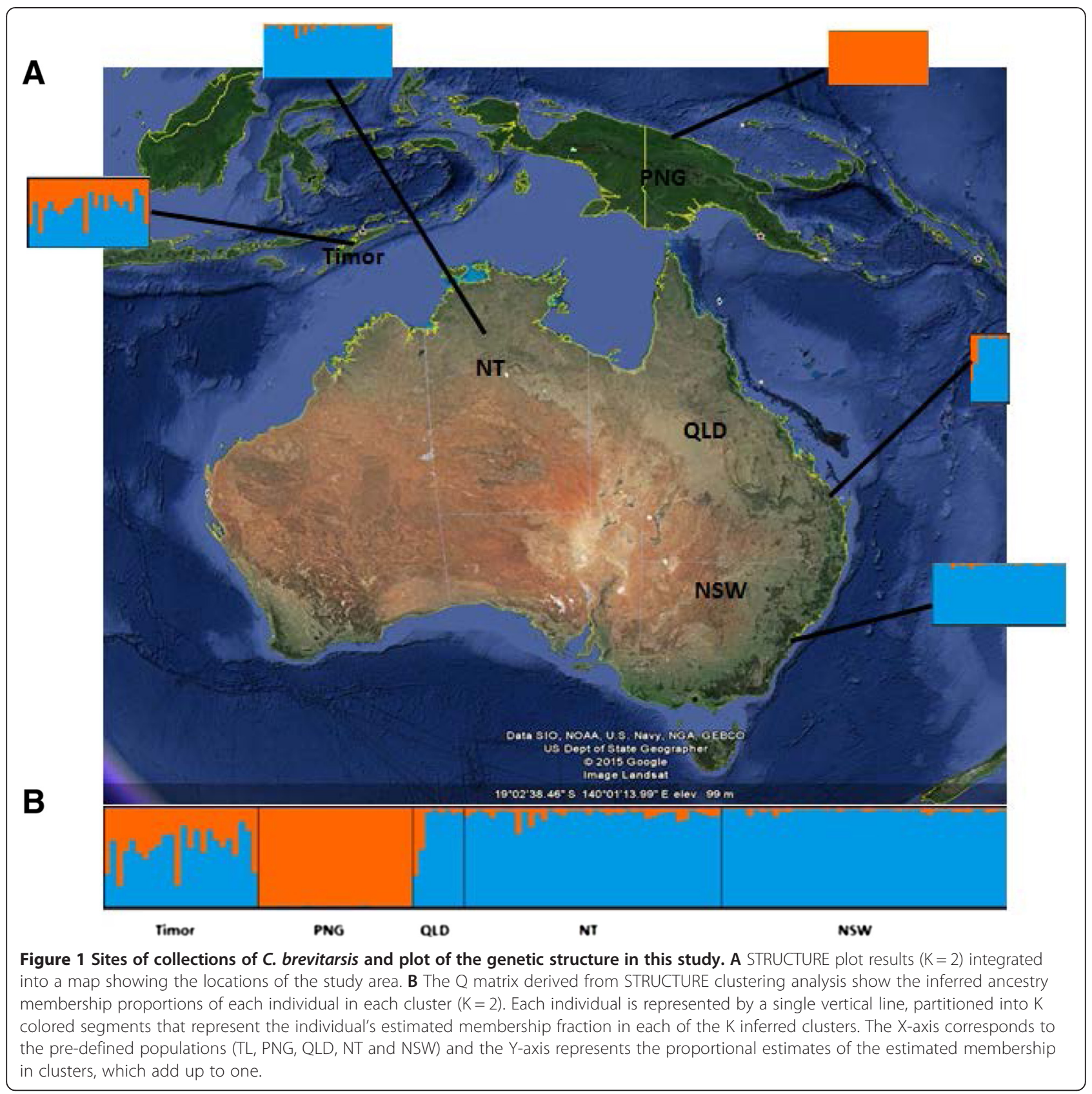

target sequences were derived from microorganisms and or other contaminants. Primers that were found to be unique i.e. neither homologous to sequences from other organisms in the database nor similar to each other were subsequently compared to our sequenced $C$. brevitarsis raw reads. The primer pairs that were predicted to anneal to more than one read were aligned to those reads after reverse-complementing the reverse primer.

\section{Microsatellite validation}

Initially, 38 primer pairs flanking dinucleotide repeats were selected for validation. Each primer pair was tested on 10 individuals. Twelve of the primer pairs that amplified $100 \%$ of the sub-set of samples (Table 1) were used to amplify DNA from 141 individuals (one individual acting as the positive control and reference allele size) sampled from three regions in Australia (Northern Territory; southeast Queensland and New South Wales) as well as from northern Papua New Guinea and Timor-Leste (Figure 1A). Each $25 \mu \mathrm{L}$ PCR reaction included $1.0 \mu \mathrm{L}$ template DNA (20 ng), $0.2 \mu \mathrm{mol}$ (each) forward and reverse fluorescently labeled primer (Applied Biosystems, USA), $18 \mu \mathrm{L}(0.396 \mathrm{U})$ of Platinum PCR supermix high fidelity (Life Technologies) and $2.75 \mu \mathrm{L}$ 
Table 1 Microsatellite loci and primers developed in this study. Locus G7B17 excluded from population genetic due to evidence of significant $(P<0.05)$ linkage to locus GO2AH

\begin{tabular}{|c|c|c|c|c|c|c|}
\hline Locus & Motif type & Range of sizes & $\mathrm{N}_{\mathrm{A}}$ & Left primer & Right primer & \#PROBEDB_ACC \\
\hline HH82P & $(A C) \wedge 13$ & $400-422$ & 13 & CACCTCTGAGAAATCCAACCG & AGTTGGTCAGCACCTCAAG & Pr032367671 \\
\hline GU21Z & $(\mathrm{GT}) \wedge 14$ & $210-222$ & 6 & TGAGTTCGTATGGCAAGGC & ACAGCGAAATGTTCATACGTG & Pr032367668 \\
\hline G7B17 & $(C A) \wedge 11$ & 204-208 & 3 & ATGGGCGAACAAATCGAGG & AACATTCGTCTTCGCTGCC & Pr032367664 \\
\hline HIIUN & $(G T) \wedge 12$ & $314-328$ & 8 & ATCCGGGAATACCTGCGAG & AAGTGTTGCCGTCGATTTC & Pr032367672 \\
\hline HNBZE & $(C A) \wedge 9$ & $328-344$ & 9 & GTGTCCGTAGCGAGTAGCC & AGCACGATTGAAACCGACAG & Pr032367673 \\
\hline G9WRZ & $(G T)^{\wedge} 8$ & $400-412$ & 6 & GCTACTGGAGCGATCTAACG & ATTAGTGTGCCGCCTTCAG & Pr032367665 \\
\hline G5L7G & $(A C) \wedge 9$ & $398-412$ & 8 & AGCATGATGAAATGTCCCGC & TCAACTACTGCTGCCCGAG & Pr032367663 \\
\hline $\mathrm{GO} 2 \mathrm{AH}$ & $(G T) \wedge 8$ & $178-194$ & 8 & TGGCTGCGAGTCGAGATG & GCCGTCGATAAGAATTAAGGTAAAC & Pr032367666 \\
\hline GONNE & $(C G G) \wedge 8$ & $304-316$ & 4 & TGATGCCCGTCCAAGATCC & GTTGCTCCGTAGTCGAACG & Pr032367667 \\
\hline G1FMO & $(A C) \wedge 11$ & $300-322$ & 7 & GCGTCATCAGTGCCAAGAC & GGAACTACACGGAGCAAGC & Pr032367662 \\
\hline $\mathrm{HBCQD}$ & $(\mathrm{GT}) \wedge 10$ & $368-386$ & 9 & GCATTTGCGTTGGCGATG & GAAGGCGTCATTCGATTTGC & Pr032367670 \\
\hline GU6HJ & $(A G) \wedge 8$ & 190-194 & 3 & GGCGATGACGATAACGAGC & ACATGACTTTGAAATTGAATCTGCC & Pr032367669 \\
\hline
\end{tabular}

de-ionized water (Life Technologies). The amplification was carried out under the following conditions: initial denaturation of $94{ }^{\circ} \mathrm{C}$ for $3 \mathrm{~min}$, then 15 cycles of $94{ }^{\circ} \mathrm{C}$ for $30 \mathrm{~s}, 60{ }^{\circ} \mathrm{C}$ for $30 \mathrm{~s}$ with a gradient decrease of $1{ }^{\circ} \mathrm{C} /$ cycle, $72{ }^{\circ} \mathrm{C}$ for $30 \mathrm{~s}$ followed by 30 cycles of $94{ }^{\circ} \mathrm{C}$ for $30 \mathrm{~s}, 45^{\circ} \mathrm{C}$ for $30 \mathrm{~s}, 72{ }^{\circ} \mathrm{C}$ for $30 \mathrm{~s}$ and a final elongation step of $72{ }^{\circ} \mathrm{C}$ for $7 \mathrm{~min}$. Amplified PCR products were fragment-sized by an external contractor (Macrogen, Geumchun-gu, Seoul). The fragment lengths were analyzed and corrected manually using Genemapper v4.0 (Applied Biosystems). A fraction of the original sample size (6\% of specimens) was re-run using touchdown PCR conditions (annealing temperature reduced to $40^{\circ} \mathrm{C}$ ) as a validation of initial allelic scores.

\section{Data analysis}

Genepop [21] was used to test for linkage disequilibrium between each pair of loci and across regions (Fischer's method) and to estimate allelic diversity and the coefficient of inbreeding $\left(\mathrm{F}_{\mathrm{IS}}\right)$ at individual loci within populations. Microchecker [22] was used to check for putative null alleles, large allele dropout or stutter peaks.

Deviations from the Hardy-Weinberg equilibrium was estimated by using Arlequin v3.0 [23]. The observed numbers of heterozygotes and homozygotes at loci in each population were tested against the expected numbers using a chi-square test.

To infer population structure from the microsatellite data, multilocus genetic distance [24] and fixation index $\left(\mathrm{F}_{\mathrm{st}}\right)$ [25] estimates were calculated between population pairs using Genepop, Arlequin and GenAlex v6.5 $[21,23,26]$. Permutation tests (100 replications) were used to determine significance of the population structure estimates.

A Bayesian clustering approach implemented in STRUCTURE v2.3.4 [27] was used to provide probabilistic estimates of population structure based on unlinked multilocus genotype distributions. Individuals are assigned probabilistically to ( $\mathrm{K}$, where $\mathrm{K}$ may be unknown) populations or jointly to two or more populations if their genotypes indicate they are admixed. The model doesn't assume a particular mutation process [27].

To generate a matrix of individual membership coefficient and population ancestry components, the following parameter set was applied: burnin period of 100000 , Markov chain Monte Carlo (MCMC) repeats of 100 000, ancestry model of admixture of LOCPRIOR model and a K value range from 1-10 with an iteration of 22 .

STRUCTURE HARVESTER v0.6.94 [28] was used to infer the most likely number of genetic clusters (K) present using both the Evanno and Delta $\mathrm{K}$ methods while CLUMPAK [29] was used to collate the data into a single matrix for all the $\mathrm{K}$ values.

\section{Results}

WGA of heat and D1 buffer-denatured DNA and quality assessment of whole genome amplified DNA

The DNA yield from WGA DNA of both heat-denatured and denaturing solution (buffer D1) denatured DNA was compared. The D1 buffer-denatured DNA yielded more WGA product than the heat-denatured DNA (data not shown). This suggests the superiority of the D1 buffer over heat as a denaturing agent in WGA. Tests using low concentration DNA sample template $(0.215 \mathrm{ng})$ resulted in higher yield of WGA products compared to yield using higher concentration DNA template (10.7 ng). The size of WGA DNA smears was from $>10 \mathrm{~kb}$ with a smear extended down to $\sim 1 \mathrm{~kb}$. The negative control reaction did not amplify. Positive controls based on actin gene amplification were successful in both the whole genome amplified heat and D1 buffer-denatured 
DNA. The negative control reaction from the same WGA reaction did not amplify.

\section{Isolation and screening of microsatellite repeats and primer design}

A total of 120005 reads of 414 average read length was obtained after sequencing the amplified whole genomic DNA. The maximum theoretical genome coverage obtained in this study was $\sim 0.25 \mathrm{X}$ (no. of reads $\mathrm{X}$ average read length/size of genome) of the approximately $200 \mathrm{MB}$ Culicoides genome [30]. A total of 2091 reads were found to contain putative microsatellite repeats. From the reads that contained putative repeats, 2594 putative microsatellite repeats were isolated (2361 dinucleotide repeats, 90 trinucleotide repeats and 98 tetranucleotides repeats). We detected approximately 52 microsatellite repeats per MB of the genome with most primers flanking AC and GT repeats (Figure 2).

A total of 526 primers were designed to the flanking regions of the microsatellite repeats. From these, 420 primers were either found to be similar to each other or homologous to sequences from other organisms in the database, while 106 primers were unique and were not homologous to anything in the NCBI database. After comparing the 106 unique primers against the sequenced reads, 93 primer pairs (Additional file 1) were predicted to anneal to a single unique read while 13 primer pairs were predicted to anneal to more than one read or to more than one site on a single read. High

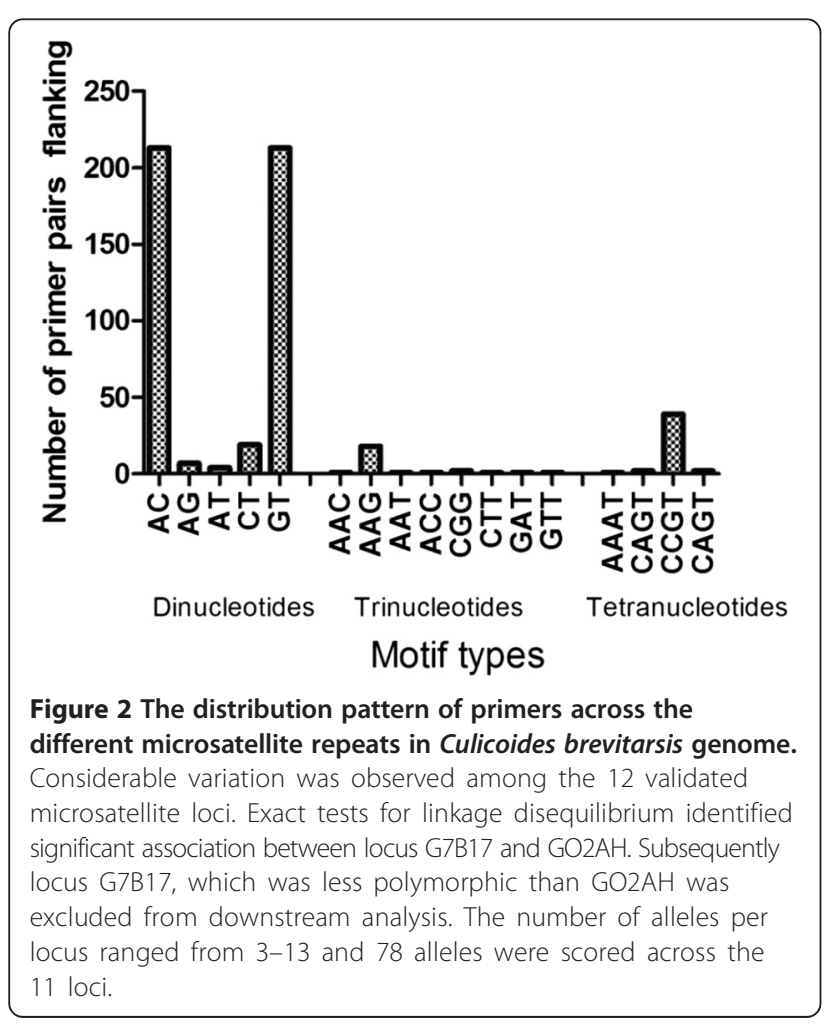

conservation between the primer pairs and the reads was evident upon aligning them to the reads. These particular primer pairs were excluded from the study to avoid multi copy amplification that may complicate the analysis procedures downstream. A subset of 38 primers was used to amplify ten individuals. Twelve of these primer pairs PCR amplified in 100\% of the individuals. The remainders amplified inconsistently and were not used further (Additional file 2).

\section{Allele frequencies distribution, heterozygosity, Hardy-Weinberg equilibrium and linkage disequilibrium} Considerable variation was observed among the 12 validated microsatellite loci (Table 1 and Additional file 3). Exact tests for linkage disequilibrium identified significant association between locus G7B17 and GO2AH. Subsequently locus G7B17, which was less polymorphic than GO2AH (Table 1) was excluded from downstream analysis. A summary of genetic diversity within the five regional populations was calculated for the remaining 11 loci (Additional file 3). The number of alleles per locus ranged from 3-13 (Table 1) and 78 alleles were scored across the 11 loci. Expected gene diversity $\left(\mathrm{H}_{\mathrm{e}}\right)$ varied from $0.01-0.89$ and in $96 \%$ of comparisons was higher than the observed heterozygosity $\left(\mathrm{H}_{\mathrm{o}}\right)$ with a range of $0-0.88$. The inbreeding coefficient $\left(\mathrm{F}_{\mathrm{IS}}\right)$ was significantly different from zero in an average of eight loci per population. Estimates of $\mathrm{F}_{\mathrm{IS}}$ differed between populations such that NT and NSW had a higher frequency of loci showing significant inbreeding compared to the other populations. Locus HBCQD was monomorphic in TL, PNG and QLD but polymorphic in NT and NSW. Global tests by locus revealed departure from Hardy-Weinberg equilibrium for most loci among all the populations. While putative null alleles were identified at some loci in some populations using Microchecker, the null alleles were not locus specific (Additional file 3) and a large proportion $(73 \%)$ of data of repeated PCRs were congruent with the initial results.

\section{Population genetic structure}

Statistically significant genetic differentiation was demonstrated between the northern PNG population and the other populations in three tests employed. Both the Nei genetic distance and $\mathrm{F}_{\mathrm{ST}}$ estimates indicated presence of population structure among all three regions. Nei's genetic distance varied from $0.03-0.31$ while the $\mathrm{F}_{\mathrm{ST}}$ estimates varied from $0.01-0.19$ (Table 2). Permutation tests in all cases indicated that genetic structure between paired regions was significant $(P<0.05)$. Paired region $\mathrm{F}_{\mathrm{ST}}$ estimates indicated the highest levels of subdivision between PNG and Australia $\left(\mathrm{F}_{\mathrm{ST}}=0.12\right)$ and between PNG and Timor-Leste $\left(\mathrm{F}_{\mathrm{ST}}=0.095\right)$. In contrast, $\mathrm{F}_{\mathrm{ST}}$ between Australia and Timor-Leste (0.03) was lower 
Table 2 Estimated genetic distance

\begin{tabular}{llllll}
\hline & TL & PNG & QLD & NT & NSW \\
\hline TL & & 0.17 & 0.14 & 0.1 & 0.09 \\
PNG & $0.09(0.0+-0.0)$ & & 0.31 & 0.27 & 0.26 \\
QLD & $0.08(0.0+-0.0)$ & $0.19(0.0+-0.0)$ & & 0.1 & 0.08 \\
NT & $0.04(0.0+-0.0)$ & $0.12(0.0+-0.0)$ & $0.06(0.0+-0.0)$ & 0.03 \\
NSW & $0.03(0.0+-0.0)$ & $0.13(0.0+-0.0)$ & $0.04(0.06+-0.02)$ & $0.01(0.08+-0.03)$ \\
\hline
\end{tabular}

Nei genetic distance values (above diagonal) and pair-wise fixation index $\left(\mathrm{F}_{\mathrm{ST}}\right)$ values (below diagonal) for sampled Australasian populations of $C$. brevitarsis. Significance level $=0.05$.

(Table 3). There was no evidence of any significant population structure between Australian populations (Table 2).

Evanno and Delta methods identified a most probable clustering value of $K=2$, for defining population group structure analysed under STRUCTURE. The clustering analysis (Figure 1B) identified inferred ancestry membership proportional probabilities of each specimen, in the optimal two cluster arrangement (Additional file 4). The posterior probability values indicate individuals from regions in Australia (NT, NSW and QLD) are of admixed ancestry with the largest proportion of their ancestry assigned to cluster 2. Timor-Leste population ancestry on the other hand is derived almost equally between cluster 1 and 2 . The PNG population is almost exclusively assigned to cluster 1 .

\section{Discussion}

Microsatellite loci are regarded as powerful molecular markers because of their high mutability, co-dominance, abundance in the genome and relative ease of scoring $[31,32]$ and have been employed to study vectors of medical and economic importance [33-37]. However, isolation of microsatellite markers from tiny and noncultivable organisms has been hampered by the limited quantity of available genomic DNA. Recently, a study by Mardulyn et al. [37] successfully isolated 10 microsatellite markers from $C$. imicola $(1-5 \mathrm{~mm})$ using recombinant approaches as described by Glenn et al. [38] and utilized the microsatellites to better understand the mechanism responsible for the northward spread of bluetongue in the Mediterranean region. In contrast, the present study isolated microsatellite markers from $C$. brevitarsis by initially increasing the amount of genomic DNA by whole genome amplification (MDA technique)

Table 3 Estimated genetic distance

\begin{tabular}{llll}
\hline Timor & PNG & AUS & \\
\hline & 0.177 & 0.082 & Timor \\
$0.10(0.0+-0.0)$ & & 0.251 & PNG \\
$0.03(0.0+-0.0)$ & $0.12(0.0+-0.0)$ & & AUS \\
\hline
\end{tabular}

Nei genetic distance values (above diagonal) and pair-wise fixation index ( $F_{\text {ST }}$ ) values (below diagonal) for sampled regional populations of $C$. brevitarsis. Significance level $=0.05$. and subsequently sequencing the whole genome amplified DNA on an eighth of a lane of a 454 GS FLX sequencer. To the best of our knowledge, this is the first study that has isolated microsatellite markers de novo from WGA DNA for any organism. The MDA-based whole genome amplification procedure provides an ideal method of isolating microsatellite markers from limited amounts of genomic DNA. This gave rise to 120005 raw reads from which 93 primer pairs that are unique to a single read (and not the products of contamination) were isolated. The abundance of microsatellite repeats may be skewed due to a potential unbalanced representation of the genome during whole genome sequencing. A selected number of markers flanking the repeats were successfully validated and utilized to study Australasian populations of $C$. brevitarsis.

Aerial arthropod dispersal modeling studies have assessed the possible routes of introduction of BTV vectors into Australia from neighboring countries including Timor-Leste and PNG. The models predict stronger pathways of dispersal from Timor-Leste to Australia, than from PNG [8,11,39]. Mitochondrial DNA (mtDNA) evidence indicates several Southeast Asian Culicoides species have entered northern Australia from both Timor-Leste and PNG [12]. More recent mtDNA evidence indicates historical pathways of dispersal by $C$. brevitarsis to northern Australia are less certain. Based on their study of COI haplotypes, Gopurenko et al. [13] found evidence of multiple independent range expansions of the species into Australasia, with evidence of separate expansions of the species into north Australia and PNG from independent Southeast Asia sources. They also identified moderate levels of gene flow in directions contrary to expectations of the historical dispersal of the species based on growth of the cattle industry in Australasia. The authors concede that gene flow estimates in their study were historical and likely influenced by migrations to the region from unsampled Southeast Asian sources.

So far, no genetic studies have used multi locus markers to examine population structure of this species. The current study applied 11 newly isolated microsatellite markers to determine the population 
genetic structure of C. brevitarsis in Timor-Leste, Australia and northern PNG.

The results here indicate populations of $C$. brevitarsis in Australia are more similar to those in Timor-Leste than in northern PNG. This is particularly evident in STRUCTURE analyses, which have assigned Australia and Timor-Leste specimens to a population cluster separate from a single PNG cluster. Pairwise $\mathrm{F}_{\mathrm{ST}}$ tests indicate significant structure exist between Australia and its northern neighbors, but the level of $\mathrm{F}_{\mathrm{ST}}$ structure evidenced between these two regions is less than that in pair-wise comparisons with PNG. The results demonstrate that $C$. brevitarsis in Australia was potentially introduced into NT from Timor-Leste or from neighboring islands in Indonesia (less likely from PNG) consistent with previous spatial modeling studies that indicated the islands of Timor and Sumba in the Indonesian Archipelago were the likely principal sources of Culicoides dispersal into northern Australia $[8,11,39]$. The gene flow barriers between northern PNG and other Australasian populations would mainly be geographic. High mountain ridges characterize PNG spatial geographical features with sharp narrow crests separated by deeply incised V-shaped valleys. The main geographical barrier to gene flow from northern PNG to Australia would be the Central and Owen Stanley Range of PNG highlands that span a distance of $200 \mathrm{~km}$ from the central cordillera and with an altitude of $4000 \mathrm{~m}$ above sea level [40]. The high peaks would result in a rough terrain that has been shown to act as an airflow barrier, which would slow dispersal of Culicoides on land [41]. In contrast, the relatively low plateau geography of Australia is likely to have given rise to the genetically homogenous population across the continent [10].

Genetic sampling of C. brevitarsis from northeastern Australia and southern PNG was not conducted in this study and future genetic work is required to examine levels of population connectivity between the two regions and to test the possibility of separate entry of BTV infected midges into northeastern Australia from the southern PNG region. The movement of other species of Culicoides from southern PNG into northern QLD has been documented [12] so movement of $C$. brevitarsis appears likely. Our starting working hypothesis was to highlight the potential larger genetic difference between the most distant NT and NSW regions and we placed more emphasis in the sampling of these two regions rather than the intermediate Queensland area. This has lead to a weaker significance of these last samples over the rest of the individuals.

Furthermore, both STRUCTURE analysis and conventional $\mathrm{F}_{\mathrm{ST}}$ estimates of population subdivision indicate C. brevitarsis populations are genetically panmictic in Australia and display no evidence of genetic separation or structure between northern and eastern sampled populations. This result suggests that the presence of the two BTV episystems in Australia (northern and eastern) as demonstrated by the distribution of BTV serotypes is not as a result of genetic structuring of this vector and is due to other factors for example, the presence of other vector species in northern Australia that are absent from southeastern Australia.

The 11 loci isolated and validated in this study were polymorphic with number of alleles ranging from 3 to 13. Departure from Hardy-Weinberg equilibrium resulting from excessive homozygote presence in one or more sampled populations was evident at all 11 loci. Severe inbreeding can cause an excess of homozygotes across loci, however, this would involve substantial and or ongoing bottlenecking of populations down to few breeding adults [42]. Substantial population bottlenecks would however have greater and immediate effect on haploid genetic markers such as maternally inherited mtDNA [43]. MtDNA evidence obtained by Gopurenko et al. [13] indicated moderate to high levels of mtDNA diversity among C. brevitarsis populations in the same regions examined in the present study and further their modeled estimates of effective maternal population size in contemporary populations was in the order of hundreds of thousands of individuals. Alternatively, null allele presence, caused by mutations at locus specific primer annealing sites which retard PCR amplification efficiency of particular alleles, can result in excesses of homozygotes scored at loci and hence significant deviation from Hardy-Weinberg Equilibrium in populations [44]. Allelic dropout in PCR caused by a variety of inhibitory causes can result in similar outcomes [45].

To validate the levels of potential allelic and genotypic miss-scores caused by a variety of PCR processes including PCR dropout and primer redundancy, a total of 86 DNA samples initially identified either as homozygous at one or more loci or had failed to amplify were re-amplified at one or more of the 11 markers using a touchdown PCR program. Three percent of samples initially identified as homozygous were scored as heterozygotes in the repeat PCRs, $17 \%$ of samples that failed to amplify previously were successfully reamplified, 7\% of samples that had been amplified before failed to amplify during this re-amplification experiment and $73 \%$ were unchanged.

The effects of null allele on test for HWE have been reported earlier [46]. The effects of null allele on genetic test of population structure vary according to the severity of the null allele presence and the type of test being used. Carlsson [47] examined effects of null allele on population structure estimated by $\mathrm{F}_{\mathrm{ST}}$ and also population assignment testing (as employed in STRUCTURE) and they identified significant but small upwards biases 
to $\mathrm{F}_{\mathrm{ST}}\left(\mathrm{F}_{\mathrm{ST}}\right.$ increased between 0.003 and 0.004) and slight reduction in the power of STRUCTURE to correctly assign individuals to populations ( 0.2 and $1.0 \%$ units). With these caveats, we argue the likely slight upward biases to our pairwise estimates of $F_{\mathrm{ST}}$ as well as some effects on specimen cluster assignment using STRUCTURE, which would not drastically change the results of our study.

We have demonstrated that whole genome amplification of the genomic DNA and subsequent whole genome sequencing resulted in a successful de novo isolation of microsatellite markers from $C$. brevitarsis. These microsatellite markers are likely to be very useful for genetically typing population origins of $C$. brevitarsis detected in the future. They can also be applied to carry out a broader analysis of gene flow in Australasian and Southeast Asian populations of C. brevitarsis.

\section{Additional files}

\section{Additional file 1: A summary file of the total number of primers} designed to the flanking regions of the microsatellite repeats. From the total primers isolated, 420 primers were either found to be similar to each other or blasted to sequences from other organisms in the database, while 106 primers were unique and did not blast to anything in the NCBI database. After blasting the 106 unique primers against the sequenced reads, 93 primer pairs were predicted to anneal to a single unique read while 13 primer pairs were predicted to anneal to more than one read or to more than one site on a single read. (XLS $36 \mathrm{~kb}$ )

Additional file 2: C. brevitarsis primer optimization. A subset of 38 primers was used to amplify 10 individuals. Twelve of these primer pairs PCR amplified $100 \%$ of the individuals. The remainders amplified inconsistently and were not used further. (XLS $28 \mathrm{~kb}$ )

Additional file 3: Summary of allelic variation in $\mathbf{1 1}$ microsatellite loci. Allelic variation among $N=141$ [Timor-Leste $(N=24)$, Papua New Guinea $(N=24)$, Queensland $(N=8)$, Northern Territory $(N=40)$ and New South Wales $(N=45)]$ C. brevitarsis specimens sampled from regional populations. Nallele- Null allele, ${ }^{\mathbf{m}}$ - monomorphic locus, observed $\left(H_{0}\right)$ and expected $\left(\mathrm{H}_{\mathrm{e}}\right)$ heterozygosity, probability of deviation from Hardy-Weinberg Equilibrium ( $\mathrm{P}_{\text {HWE }}$ ) and inbreeding co-efficient $\left(F_{I S}\right)$ as described. Significantly deviated $F_{15}$ values $(P<0.05)$ highlighted in BOLD type. (XLS $38 \mathrm{~kb}$ )

Additional file 4: Probability values of population assignments for samples $\mathrm{K}=\mathbf{2}$. The population $\mathrm{Q}$ matrix derived from STRUCTURE clustering analysis show the inferred ancestry membership proportions of each individual, in each cluster. Each individual is represented by a single vertical line, partitioned into $\mathrm{K}$ colored segments that represents that individual's estimated membership fraction in each of the $\mathrm{K}$ inferred clusters. The $\mathrm{X}$ axis corresponds to the pre-defined populations (TL, PNG, QLD, NT and NSW and the $Y$ axis represents the proportional estimates of the estimated membership in clusters which add up to one. (TXT $11 \mathrm{~kb}$ )

\section{Competing interests}

The authors declare that they have no competing interests.

\section{Authors' contributions}

MGO designed, conducted the experiments, analyzed results and wrote the paper. NWB participated in the microsatellite development and experimental design. DG participated in the writing of the paper, provided extracted DNA from Timor and PNG and participated in the study design. GB did the sampling, specimen identification and participated in the study design. AN did the sampling, specimen identification and participated in the study design. $\mathrm{MO}$ participated in data analysis. AD and SK participated in the study design.
PJW involved in the study design, participated in the writing of the paper. $J \mathrm{BD}$ involved in the study design, experimental design and participated in the writing of the paper. All authors read and approved the final manuscript.

\section{Acknowledgements}

M.G. Onyango was supported by a scholarship from the Australian government. Laboratory works at AAHL have been partly performed thanks to the National Collaborative Research Infrastructure Strategy of Australia. The authors gratefully acknowledge David Boyle and Rhonda Voysey for informative discussions. We would like to thank Peter Mee for providing us with samples of C. brevitarsis from Queensland. Our gratitude also goes to Johnson Mak (CSIRO, AAHL) and George N. Michuki (International Livestock Research Institute) for incisive suggestions and discussions. We acknowledge Cadhla Firth and Debbie Eagles (both CSIRO, AAHL) for the critical review of the manuscript. We thank PNG NAQIA, Timor Leste MAF and the Australian National Arbovirus Monitoring Program for access to specimens and permission to publish data from their respective countries.

\section{Author details}

${ }^{1}$ CSIRO Health \& Biosecurity Australian Animal Health Laboratory, 5 Portalington Road, Geelong, Victoria 3220, Australia. ${ }^{2}$ School of Medicine, Deakin University, 75 Pidgons Road, Waurn Ponds, Victoria 3216, Australia. ${ }^{3}$ School of Biological Sciences, The University of Queensland, St Lucia, Queensland 4072, Australia. ${ }^{4}$ CSIRO Health \& Biosecurity Ecosciences Precinct, 41, Boggo Road, Dutton Park, Queensland 4102, Australia. ${ }^{5}$ NSW Department of Primary Industries, Wagga Wagga Agricultural Institute, PMB, Wagga Wagga, New South Wales 2650, Australia. ${ }^{6}$ Graham Centre for Agricultural Innovation, Locked Bag 588, Wagga Wagga, New South Wales 2678, Australia. ${ }^{7}$ Northern Australia Quarantine Strategy, 1 Pederson Road, Marrara, Northern Territory 0812, Australia. International Livestock Research Institute, P.O. Box 3070900100 Nairobi, Kenya. ${ }^{9}$ Biosciences eastern and central Africa ILRI Hub (BecA-ILRI Hub), ILRI, PO Box 3070900100 Nairobi, Kenya.

Received: 3 June 2015 Accepted: 24 August 2015

Published online: 25 September 2015

\section{References}

1. George S (1989) An overview of arboviruses affecting domestic animals in Australia. Aust Vet J 66:393-395

2. Mellor PS, Boorman J, Baylis M (2000) Culicoides biting midges: their role as arbovirus vectors. Annu Rev Entomol 45:307-340

3. Bellis GA, Dyce AL (2005) Intraspecific variation in three species of Culicoides in the Orientalis complex of the subgenus Avaritia within Australiasian zoogeographic region. In Arbovirus research in Australia: papers from $9^{\text {th }}$ Arbovirus Research in Australia Symposium, $6^{\text {th }}$ Mosquito control Association of Australia Symposium, 22-27 August 2004, Australis Noosa Lakes Resort, Australia eds PA Ryan, JG Askov, TD St George, PER Dale, The Queensland Institute of Medical Research, Brisbane, Australia 9:33-36

4. Standfast HA, Dyce AL, Muller MJ (1985) Vectors of bluetongue virus in Australia. Prog Clin Biol Res 178:177-186

5. Muller MJ (1995) Veterinary arbovirus vectors in Australia - a retrospective. Vet Microbiol 46:101-116

6. Ward MP (1996) Seasonality of infection of cattle with bluetongue viruses. Prev Vet Med 26:133-141

7. Dyce AL (1982) Distribution of Culicoides (Avaritia) species (Diptera: Ceratopogonidae) west of the Pacific ocean. In: St. George TD, Kay BH (eds) Arbovirus research in Australia: proceedings $3^{\text {rd }}$ symposium, 15-17 February 1982. CSIRO and QIMR, Brisbane, pp 35-43

8. Eagles D, Melville L, Weir R, Davis S, Bellis G, Zalucki MP, Walker PJ, Durr PA (2014) Long-distance aerial dispersal modelling of Culicoides biting midges: case studies of incursions into Australia. BMC Vet Res 10:135

9. Boyle DB, Bulach DM, Amos-Ritchie R, Adams MM, Walker PJ, Weir R (2012) Genomic sequences of Australian bluetongue virus prototype serotypes reveal global relationships and possible routes of entry into Australia. J Virol 86:6724-6731

10. St George TD, Baldock C, Bellis G, Bishop A, Cameron A, Doherty B, Ellis T, Gard G, Johnson S, Kirkland P, Melville L, Muller M, Postle T, Roe D (1999) The history of Bluetongue, Akabane and ephemeral fever viruses and their vectors in Australia, National Arbovirus Monitoring (NAMP) Report 
11. Eagles D, Deveson T, Walker PJ, Zalucki MP, Durr P (2012) Evaluation of long-distance dispersal of Culicoides midges into northern Australia using a migration model. Med Vet Entomol 26:334-340

12. Bellis GA, Gopurenko D, Cookson B, Postle AC, Halling L, Harris N, Yanase T, Mitchell A (2015) Identification of incursions of Culicoides Latreille species (Diptera: Ceratopogonidae) in Australasia using morphological techniques and DNA barcoding. Austral Entomol 54:332-338

13. Gopurenko D, Bellis G, Mitchell A (2011) Integrative taxonomic assessment and DNA barcoding of economically important biting midges (Ceratopogonidae: Culicoides) present in Australasia. In: Fourth International Barcode of Life Conference Adelaide Australia 2011., http://www.dnabarcodes2011.org

14. Avise JC (1991) Ten unorthodox perspectives genetic findings on evolution prompted by comparative population genetic findings on mitochondrial DNA. Annu Rev Genet 25:46-69

15. Roderick GK (1996) Geographic structure of insect populations: gene flow, phylogeography, and their uses. Annu Rev Entomol 41:325-352

16. Bellis GA, Dyce AL, Gopurenko D, Mitchell A (2013) Revision of the Immaculatus Group of Culicoides Latreille (Diptera: Ceratopogonidae) from the Australasian Region with description of two new species. Zootaxa 3680:15-37

17. Bellis GA, Dyce AL, Gopurenko D, Yanase T, Garros C, Labuschagne K, Mitchell A (2014) Revision of the Culicoides (Avaritia) Imicola complex Khamala \& Kettle (Diptera: Ceratopogonidae) from the Australasian region. Zootaxa 3768:401-427

18. Staley M, Dorman KS, Bartholomay LC, Farfan-Ale JA, Loroño-pino MA, Julian E, Ibarra-juarez L, Blitvich BJ (2010) Sequence analysis of Actin-1 from diverse mosquito species. J Am Mosq Control Assoc 26:214-218

19. Faircloth BC (2008) Msatcommander: detection of microsatellite repeat arrays and automated, locus-specific primer design. Mol Ecol Resour 8:92-94

20. Koressaar T, Remm M (2007) Enhancements and modifications of primer design program Primer3. Bioinformatics 23:1289-1291

21. Rousset F (2007) genepop'007: a complete re-implementation of the genepop software for Windows and Linux. Mol Ecol Resour 8:103-106

22. Van Oosterhout C, Hutchinson WF, Wills DPM, Shipley P (2004) MicroChecker: software for identifying and correcting genotyping errors in microsatellite data. Mol Ecol Notes 4:535-538

23. Excoffier L, Laval G, Schneider S (2007) Arlequin (version 3.0): an integrated software package for population genetics data analysis. Evol Bionform Online 1:47-50

24. Nei M (1978) Estimation of average heterozygosity and genetic distance from a small number of individuals. Genetics 89:583-590

25. Weir BS, Cockerham CC (1984) Estimating F-statistics for the analysis of population structure. Evolution 38:1358-1370

26. Peakall R, Smouse PE (2012) GenAlEx 6.5: genetic analysis in Excel. Population genetic software for teaching and research-an update. Bioinformatics 28:2537-2539

27. Pritchard JK, Stephens M, Donnelly P (2000) Inference of population structure using multilocus genotype data. Genetics 155:945-959

28. Evanno G, Regnaut S, Goudet J (2005) Detecting the number of clusters of individuals using the software STRUCTURE: a simulation study. Mol Ecol $14: 2611-2620$

29. Kopelman NM, Mayzel J, Jakobsson M, Rosenberg NA, Mayrose I (2015) Clumpak : a program for identifying clustering modes and packaging population structure inferences across K. Mol Ecol Resour 15:1179-1191

30. Nayduch D, Cohnstaedt LW, Saski C, Lawson D, Kersey P, Fife M, Carpenter S (2014) Studying Culicoides vectors of BTV in the post-genomic era: resources, bottlenecks to progress and future directions. Virus Res 182:43-49

31. Lagoda PJL (1996) Microsatellites, from molecules to populations and back Trends Ecol Evol 11:424-429

32. Tóth G, Gáspári Z, Jurka J (2000) Microsatellites in different eukaryotic genomes: survey and analysis. Genome Res 10:967-981

33. Lehmann T, Hawley WA, Grebert H, Collins FH (1998) The effective population size of Anopheles gambiae in Kenya : implications for population structure. Mol Biol Evol 15:264-276

34. Lehmann T, Hawley WA, Kamau L, Fontenille D, Simard F, Collins FH (1996) Genetic differentiation of Anopheles gambiae populations from East and West Africa : comparison of microsatellite and allozyme loci. Heredity (Edinb) 77:192-200

35. Oliveira R, Broude N, Macedo A, Cantor C, Smith C, Pena D (1998) Probing the genetic population structure of Trypanosoma cruzi with polymorphic microsatellites. Proc Natl Acad Sci U S A 95:3776-3780
36. Ravel S, Monteny N, Olmos DV, Verdugo JE, Cuny G (2001) A preliminary study of the population genetics of Aedes aegypti (Diptera: Culicidae) from Mexico using microsatellite and AFLP markers. Acta Trop 78:241-250

37. Mardulyn P, Goffredo M, Conte A, Hendrickx G, Meiswinkel R, Balenghien T, Sghaier S, Lohr Y, Gilbert M (2013) Climate change and the spread of vector-borne diseases: using approximate Bayesian computation to compare invasion scenarios for the bluetongue virus vector Culicoides imicola in Italy. Mol Ecol 22:2456-2466

38. Glenn TC, Schable N (2005) Isolating microsatellite DNA loci. Methods Enzymol 395:202-222

39. Eagles D, Walker PJ, Zalucki MP, Durr PA (2013) Modelling spatio-temporal patterns of long-distance Culicoides dispersal into northern Australia. Prev Vet Med 110:312-322

40. Loffier E (1982) Landforms and landform development. In Biogeogr Ecol New Guinea. Edited by JL. Gressitt, Dr W. Junk Publishers The Hague pp 57-72.

41. Hendrickx G, Gilbert M, Staubach C, Elbers A, Mintiens K, Gerbier G, Ducheyne E (2008) A wind density model to quantify the airborne spread of Culicoides species during north-western Europe bluetongue epidemic. Prev Vet Med 87:162-181

42. Nei M, Maruyama T, Chakraborty R (1975) The bottleneck effect and genetic variability in populations. Evolution 29:1-10

43. Wilson AC, Cann RL, Carr SM, George M, Gyllensten UB, Helm-Bychowski KM, Higuchi RG, Palumbi SR, Prager EM, Sage RD, Stoneking M (1985) Mitochondrial DNA and two perspectives on evolutionary genetics. Biol J Linn Soc 26:375-400

44. Kelly AC, Mateus-Pinilla NE, Douglas M, Douglas M, Shelton P, Novakofski J (2011) Microsatellites behaving badly: empirical evaluation of genotyping errors and subsequent impacts on population studies. Genet Mol Res 10:2534-2553

45. Séré M, Kaboré J, Jamonneau V, Belem AM, Ayala FJ, De Meeûs T (2014) Null allele, allelic dropouts or rare sex detection in clonal organisms: simulations and application to real data sets of pathogenic microbes. Parasit Vectors 7:331

46. Nascimento de Sousa S, Finkeldey R, Gailing O (2005) Experimental verification of microsatellite null alleles in Norway spruce (Picea abies [L.] Karst.): implications for population genetic studies. Plant Mol Biol Report 23:113-119

47. Carlsson J (2008) Effects of microsatellite null alleles on assignment testing. J Hered 99:616-623

\section{Submit your next manuscript to BioMed Central and take full advantage of:}

- Convenient online submission

- Thorough peer review

- No space constraints or color figure charges

- Immediate publication on acceptance

- Inclusion in PubMed, CAS, Scopus and Google Scholar

- Research which is freely available for redistribution

Submit your manuscript at www.biomedcentral.com/submit
C BioMed Central 